\title{
A IMPORTÂNCIA DO SISTEMA DE GESTÃO AMBIENTAL PARA OBTENÇÃO DE SELOS SUSTENTÁ VEIS NA CONSTRUÇÃO CIVIL: UMA REVISÃO NARRATIVA
}

THE IMPORTANCE OF THE ENVIRONMENTAL MANAGEMENT SYSTEM FOR OBTAINING SUSTAINABLE SEALS IN CIVIL CONSTRUCTION: A NARRATIVE REVIEW

Ana Claudia Marangoni Batista Campana ${ }^{1}$; Dariane dos Santos Virgens Alvarenga da Silva $^{2}$; Josiane Marlise Theis Aguirre ${ }^{3}$, Letícia dos Santos Marangoni Sígoli4; Máriam Trierveiler Pereira ${ }^{5}$.

1. Mestranda do Programa de Pós-Graduação em Sustentabilidade - Instituto Federal do Paraná (IFPR), Campus Umuarama, bolsista PIBPós IFPR, marangoni.arq@ gmail.com.

2. Mestranda do Programa de Pós-Graduação em Sustentabilidade - Instituto Federal do Paraná (IFPR), Campus Umuarama.

3. Mestranda do Programa de Pós-Graduação em Sustentabilidade - Instituto Federal do Paraná (IFPR), Campus Umuarama.

4. Mestranda do Programa de Pós-Graduação em Rede Nacional para Ensino das Ciências Ambientais (PROFCIAMB) - Universidade Estadual de Maringá (UEM), Campus Maringá.

5. Professora do Programa de Pós-Graduação em Sustentabilidade - Instituto Federal do Paraná (IFPR), Campus Umuarama.

\section{RESUMO}

A certificação ambiental para edifícios (CAE) surgiu como forma de impulsionar o ramo das construções sustentáveis, pois a construção civil causa grandes impactos ambientais negativos. Sabe-se que a implantação das certificações exige um Sistema de Gestão Ambiental (SGA). O objetivo do trabalho foi fazer uma revisão narrativa sobre a importância do SGA para a CAE. Utilizou-se do método de revisão bibliográfica, abordando-se a definiçãa de SGA, com foco na NBR ISO 14001 (2015), e comparandose o selo AQUA, LEED e Casa Azul. A certificação AQUA demonstrou ser mais criteriosa. Em análise de um estudo de caso entre dois empreendimentos que buscaram o selo AQUA, um na fase de planejamento e outro após o início da construção, verificouse que o SGA implantado desde o planejamento da obra apresentou melhores resultados na certificação. Conclui-se que o SGA demonstrou ser um investimento viável devido a várias vantagens que proporciona ao longo da obra.

\section{Palavras Chave}

Certificação de edifícios; Construções sustentáveis; Gerenciamento de obras; NBR ISO 14001; Certificação ambiental. 


\section{ounisul}

\section{ABSTRACT}

The buildings environmental certification (BEC) emerged as a way to promote the sustainable building, because civil construction causes great negative environmental impacts. We known that the certification implementation requires an Environmental Management System (EMS). The aim of this work was to make a narrative review about the importance of the EMS for the (BEC). We used the literature review method to EMS definition with focus in NBR ISO 14001 (2015), and we compared the AQUA, LEED and Casa Azul green seals. The AQUA certification proved to be more discerning. We also analysed a case study between two projects with AQUA seal, one in the planning phase and the other post-start construction phase. We founded that the EMS implemented since the planning phase presented better results in terms of certification. We concluded that the EMS proved to be a viable investment due to several advantages it supply throughout the construction.

\section{KEY WORDS}

Green building certification; Sustainable building; Construction management; NBR ISO 14001; Environmental certification

\section{INTRODUÇÃO}

A exploração de recursos naturais do planeta tem chamado atenção em todos os setores da sociedade nos últimos anos. A construção civil fica em evidência nesse cenário por ser um dos setores que mais geram resíduos e afetam o meio ambiente e alteram a paisagem urbana, produzindo grandes impactos negativos que vão desde a retirada das matériasprimas da natureza, até a destinação final dos resíduos (BARRETO, 2005). Heywood (2017) afirma que as grandes expansões dos centros urbanos aumentaram o impacto negativo sobre o meio ambiente. Por esse motivo, a implantação de edifícios sustentáveis tornou-se necessária.

Um empreendimento sustentável é aquele que atende os três pilares da sustentabilidade, social, econômico e ambiental. O pilar social é alcançado por meio de geração de empregos, integração entre os usuários e a vizinhança, e pagamento de impostos que são convertidos em benefícios para a população; já o pilar econômico provém do retorno financeiro justo para os empreendedores e usuários, uso racional dos materiais, e aumento da produtividade dos trabalhadores devido à disponibilidade de um ambiente de trabalho saudável e seguro; quanto ao pilar ambiental, este é atingindo quando há uma otimização dos materiais, geração de menos de resíduos, menor uso de 
energia e água, menor desmatamento de áreas verdes, além da produção de obras duráveis, flexíveis, que possa ser adequadas e requalificadas conforme a necessidade (MAGDALENO; NÓBREGA, 2016).

Nessa linha de raciocínio, as Certificações Ambientais para Edifícios, também chamados de Selos Verdes, surgiram como forma de incentivar a construção de edificações sustentáveis. Porém, para alcançá-las é necessário obedecer a metodologia que cada tipo de selo propõe.

As metodologias são diversas, entretanto para pôr em prática qualquer uma delas, é necessário um Sistema de Gestão Ambiental (SGA) integrado à obra, pois assim todos os processos da edificação são organizados. Essas metodologias surgiram na década de 90 na Europa e na América do Norte como tática para cumprir as metas estabelecidas na ECO-92, a fim de estimular o desempenho ambiental da indústria da construção civil (SILVA; SILVA; AGOPYAN, 2003).

No presente trabalho será abordada a definição da gestão ambiental para edificações e qual sua influência para a obtenção de certificações sustentáveis, avaliando sua viabilidade, com as vantagens e desvantagens do processo. A justificativa para esse estudo é a importância do tema da construção civil sustentável, que merece ser mais explorado com o desejo de popularizar o SGA e também a certificação ambiental de edifícios.

Portanto, o objetivo deste trabalho foi realizar uma revisão narrativa a respeito da importância do SGA para a obtenção de Selo de Certificação Ambiental na Construção Civil.

\section{MATERIAIS E MÉTODOS}

Trata-se de uma pesquisa qualitativa de método indutivo, por meio da revisão bibliográfica de autores que abordam sobre o tema.

O desenvolvimento do trabalho traz três assuntos: sistema de gestão ambiental na construção civil; certificações ambientais para edificações; e o papel da gestão ambiental 


\section{ounisul}

para obtenção de selos ambientais em edifícios. O primeiro subtítulo aborda o SGA, do que se trata, sua importância, vantagens e desvantagens; o segundo trata sobre as certificações ambientais para a construção civil, como funciona, métodos de procedimento, cita os principais sistemas de certificação e compara seus critérios de obtenção; já o terceiro explora como a gestão ambiental influencia nesse processo de certificação.

Também foi utilizado do método de procedimento comparativo por meio da construção de um quadro dos critérios exigidos para obtenção dos selos de sustentabilidade, comparando três dos principais sistemas de certificação utilizados no Brasil: LEED, AQUA e Casa Azul CAIXA. Para a elaboração desse quadro foram explorados os checklists disponíveis nos portais eletrônicos dos sistemas analisados e descreveu-se os critérios de acordo com as semelhanças entre eles.

\section{DESENVOLVIMENTO}

A preocupação com fim dos recursos naturais do planeta é cada vez mais evidente, projetando-se em todos os segmentos, inclusive na construção civil. Magdaleno e Nóbrega (2016) afirmam que a conscientização ambiental e o conceito de projeto ecológico surgiram na década de 1990, a fim de diminuir os impactos adversos da construção civil no meio ambiente e melhorar a eficiência energética das edificações. Segundo Ramos et al. (2015), a construção civil consome recursos naturais, modifica a paisagem e gera diversos resíduos, ou seja, embora seja umas das principais atividades de crescimento socioeconômico é também uma grande causadora de impactos ambientais.

Como forma de incentivo para minimizar esses impactos ambientais, foram criados sistemas de selos verdes, cuja intenção é impulsionar o melhoramento do ambiente tanto por questões ambientais quanto por questões de competitividade do mercado (GRÜNBERG et al., 2014). Porém, para alcançar as certificações desejadas, é necessário obedecer a uma série de requisitos, entre eles a gestão ambiental do empreendimento. 


\section{ounisul}

\subsection{Sistema de Gestão Ambiental na Construção Civil}

De acordo com a Associação Brasileira de Normas Técnicas (ABNT, 2015, p. 14), pode-se definir o termo Sistema de Gestão Ambiental como "parte do sistema de gestão [...] usado para gerenciar aspectos ambientais [...], cumprir requisitos legais e outros requisitos [...], e abordar riscos e oportunidades [...]."

Assim, o SGA pode ser utilizado em diversos segmentos empresariais, sua implantação é baseada em uma série metodológica de rotinas e procedimentos que visam gerenciar todas as etapas do processo, desde o projeto, aquisição de matéria prima, e execução da proposta (VECHI; GALLARDO; TEIXEIRA, 2016).

Dal Forno (2017) afirma que devido às preocupações com o meio ambiente, o gerenciamento ambiental tornou-se um importante instrumento que produz mudanças produtivas de consumo e comercialização em todos os setores, inclusive no segmento da construção civil. Dessa forma, as instituições estão investindo em tecnologia voltada à Gestão Ambiental, a fim de implantar o processo em suas obras e expor a contribuição com a proteção do meio ambiente (RAMOS et al., 2015). Afinal, segundo os autores, "um SGA bem estruturado possibilitará organizar e desenvolver procedimentos e recursos para manter uma política ambiental e praticar suas atividades normalmente sem perder a produtividade" (RAMOS et al., 2015, p.2).

Para que haja a implantação de um SGA em uma edificação é necessário cumprir os requisitos pré-estabelecidos na NBR ISO 14001: 2015 - Sistemas da gestão ambiental: requisitos com orientações para uso. Assim como outras normas de gestão, esta diretriz classifica o SGA em um ciclo de 4 fases, denominado Ciclo PDCA (Plan-Do-Check-Act): planejar - estabelecer planos de acordo com os objetivos almejados; fazer - colocar os planos em prática; checar - monitorar a execução desses planos de acordo com as políticas ambientais e objetivos da empresa; e agir - atuar em busca do avanço da melhoria alcançada (ABNT, 2015).

A Figura 1 apresenta o Ciclo PDCA, que inicia com o planejamento. Nesse processo são expostas as necessidades e desejos dos interessados no empreendimento. Em seguida 


\section{DUnisul}

vem a fase de realização, onde começa a aparecer os resultados pretendidos do SGA e logo após é realizada a checagem de tudo que já foi feito e também a análise dos resultados obtidos. Com a checagem realizada é necessário agir para pôr em prática o que foi idealizado, e então avaliam-se os problemas internos e externos que surgiram e voltase ao planejamento para solucionar tais problemas. $\mathrm{O}$ ciclo deve continuar até a finalização do SGA.

Figura 1: Adaptação da relação entre o ciclo PDCA e a estrutura da NBR ISO 14001: 2015.

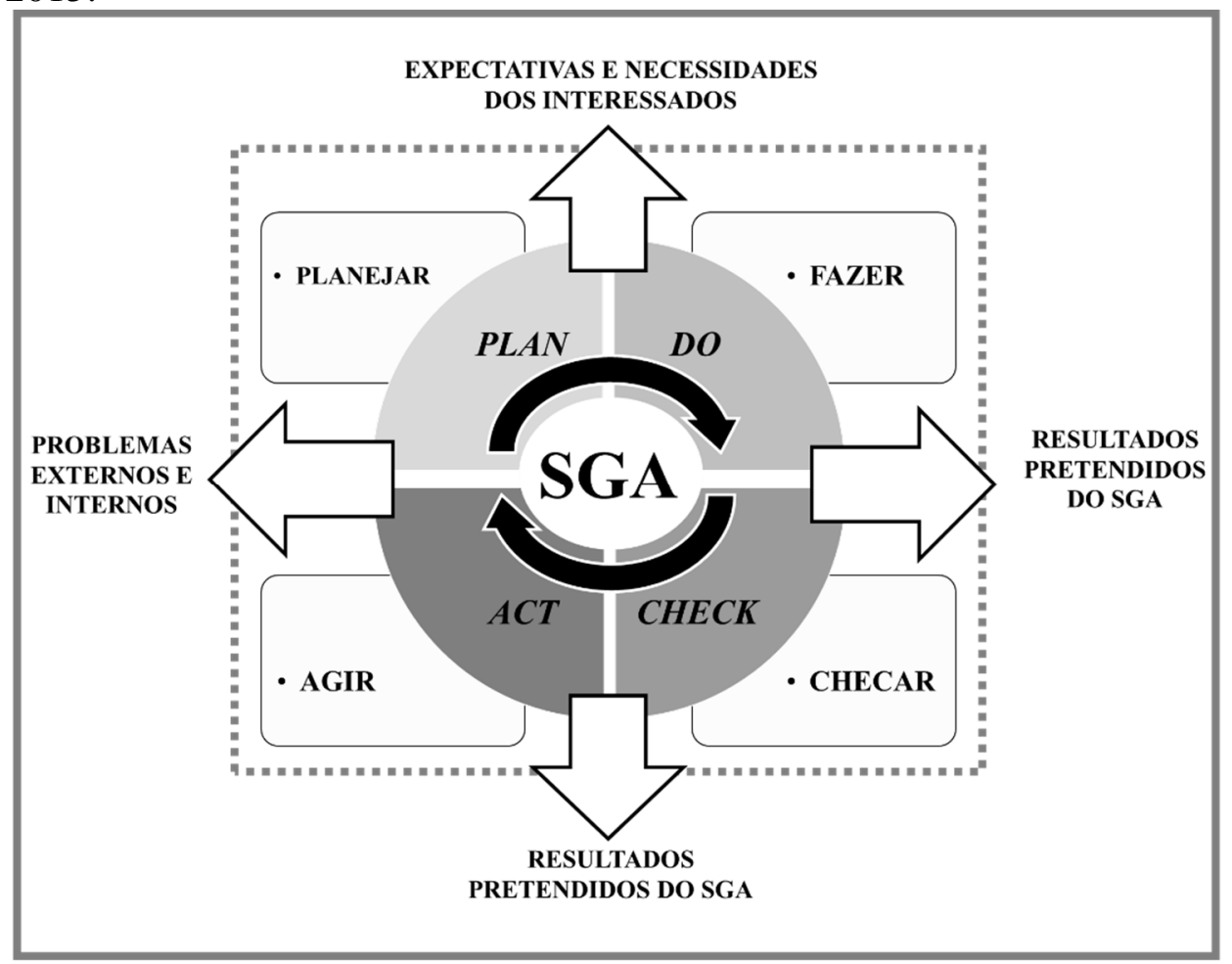

Fonte: ABNT (2015), modificado pelas autoras (2021).

Portanto, o SGA integra todas as etapas de uma obra, desde seu planejamento e concepção. Há ainda que considerar a organização que a implantação do SGA proporciona nas obras de construção civil, desde a elaboração dos projetos ao canteiro de obra (VECHI; GALLARDO; TEIXEIRA, 2016).

De acordo com a ABNT (2015), o SGA deve organizar os produtos e serviços utilizados pelo empreendimento, selecionar os aspectos ambientais que devem ser considerados e classificá-los em categorias, levando em consideração o empreendimento 


\section{ounisul E}

como um todo. Ainda segundo o mesmo autor, esses aspectos ambientais podem ser categorizados como: emissões para o ar; lançamentos em água; lançamentos em terra; uso de recursos naturais e matérias-primas; uso de energia; emissão de energia; geração de subprodutos e/ou rejeito; e uso do espaço.

Porém, essas categorias são variáveis de acordo com o empreendimento, "[...] é a organização que determina a extensão de controle que ela é capaz de exercer, os aspectos ambientais que ela pode influenciar e a extensão para os quais ela escolhe exercer tal influência" (ABNT, 2015, p. 27). Portanto, diante do SGA, cabe aos responsáveis do empreendimento determinarem como executarão sua interferência no meio ambiente.

Embora haja um crescimento da demanda de SGA na construção civil, no Brasil ainda há uma certa rejeição, pois é visto como uma mera formalização para obtenção de uma certificação, com um custo adicional dos serviços prestados para sua implantação. Porém, estudos mostram que a viabilidade é compensada pelos diversos benefícios gerados por uma obra sustentável (VECHI; GALLARDO; TEIXEIRA, 2016).

Para Barreto (2005), entre os benefícios alcançados pelo SGA, podem-se destacar ações que minimizam os impactos ambientais gerados pelos rejeitos da construção civil (entulhos), além de proporcionar benefícios de ordem ambiental, econômica e social, como: uso racional de água e energia elétrica, utilização de materiais mais sustentáveis, redução de desperdícios, entre outros. Ramos et al. (2015) afirmam ainda que a implementação de um SGA provém obtenção de recursos para a contínua melhoria dos processos, visto que o sucesso de uma política ambiental promove a redução de retrabalhos, perdas e absenteísmo.

Gomes et al. (2016) asseguram que grande parte dos materiais utilizados na construção civil provem dos recursos naturais, como a madeira, ferro, gesso, areia, cimento, brita etc. O SGA controla o uso desses materiais e evita desperdícios, além de planejar as etapas do empreendimento, fiscalizar a execução dos serviços, evitar retrabalhos e esbanjamento de matéria prima, e promover a utilização eficiente do tempo no canteiro de obras (GOMES et al., 2016). 


\section{ounisul}

Segundo Oliveira, Sposto e Blumenschein (2012), o SGA tende a se popularizar na construção civil, visto que aumenta o sucesso empresarial e a competitividade entre as empresas, logo produz resultados tangíveis e intangíveis, sendo os tangíveis relacionados a eficiência operacional das edificações, e as intangíveis, a reputação da empresa e valorização da marca.

A implantação de um SGA integrada entre todos os setores, desde o início do projeto até a entrega da obra, gera mais vantagens que desvantagens, visto que o investimento retorna à empresa por meio de redução de geração de resíduos, redução de consumo de materiais, além de proporcionar melhores condições socioambientais (GOMES et al., 2016).

Portanto, embora a implantação do SGA gere um custo inicial, o processo todo gera economia, pois obtém-se maior organização e controle de processos.

\subsection{Certificações Ambientais para edificações}

Na década de 1980, o aspecto de desempenho ambiental no processo de concepção de projeto arquitetônico já recebia atenção, intensificando-se na interpretação da Agenda 21, que influenciou todos os setores da sociedade, dando origem ao Sistema de Certificação de Desempenho Ambiental de Edifícios (BUENO; ROSSIGNOLO, 2010).

De acordo com Grünberg et al. (2014), as certificações ambientais, ou selos verdes, foram exploradas por diversos países, cada uma com uma metodologia de certificação diferente. Entre os principais selos encontram-se: o da Inglaterra - Building Research Establishment (BRE); o dos Estados Unidos da América - Liderança em Design de Energia e Meio Ambiente (LEED); o da França - Alta Qualidade Ambiental (HQE); o do Japão - Sistema de Avaliação de Eficiência Ambiental para Construção (CASBEE); e o Desafio da Construção Sustentável (GBC) que nasceu de um conjunto de vários países (GRÜNBERG et al., 2014).

Já no Brasil, podem-se destacar o uso dos seguintes selos: o Alta Qualidade Ambiental (AQUA), processo de certificação brasileiro criado pela Fundação Vanzolini baseado na 


\section{ounisul}

metodologia do francês HQE; a etiquetagem do Programa Nacional de Conservação de Energia Elétrica (PROCEL Edifica) e do Instituto Nacional de Metrologia, Qualidade e Tecnologia (INMETRO), que avaliam o grau de eficiência energética dos edifícios; o norte-americano LEED, difundido em vários países; e o Casa Azul CAIXA, criado pela Caixa Econômica Federal (ASBEA, 2012).

Como mostram Silva, Silva e Agopyan (2003), os métodos de avaliação são diferentes para cada sistema de certificação devido aos locais de origem (pois cada país sofre uma pressão ambiental diferente) e às práticas construtivas de cada região, além do índice socioeconômico de cada local. Porém, todos foram planejados sobre uma mesma base, a diminuição dos impactos ambientais (SILVA; SILVA; AGOPYAN, 2003).

Portanto, os métodos aplicados por cada um desses selos variam conforme os objetivos almejados, podendo ser avaliados de acordo com os aspectos ambientais, sociais e econômicos (ASBEA, 2012).

No estudo de Silva, Silva e Agopyan (2003), os autores analisaram vários sistemas internacionais de certificação de edifícios e identificaram que a maioria atende algumas categorias de avaliação em comum, sendo elas: estratégia de implantação, uso de água, uso de energia, materiais e resíduos, prevenção de poluição, qualidade ambiente interno, qualidade dos serviços, desempenho econômico e processo de gestão ambiental.

Com base no estudo de Silva, Silva e Agopyan (2003), foi elaborada uma comparação entre os critérios de três sistemas de certificação de edifícios sustentáveis frequentemente utilizados no Brasil, o LEED, o AQUA e o selo Casa Azul CAIXA. O Quadro 1 mostra essa análise comparativa. Os critérios semelhantes foram sobrepostos, por exemplo, o LEED utiliza o termo "eficiência hídrica" e o Casa Azul CAIXA utiliza "gestão eficiente da água". Como ambos possuem as mesmas características, a termo usado no Quadro 1 foi "uso eficiente da água". 
Quadro 1: Comparação de critérios de pontuação dos sistemas de certificações de edifícios sustentáveis

\begin{tabular}{|l|c|c|c|}
\hline Critério & LEED & AQUA & $\begin{array}{c}\text { Casa Azul } \\
\text { CAIXA }\end{array}$ \\
\hline Localização, transporte e qualidade urbana do entorno & $\mathrm{X}$ & $\mathrm{X}$ & $\mathrm{X}$ \\
\hline Terrenos sustentáveis & $\mathrm{X}$ & & \\
\hline Uso eficiente da água & $\mathrm{X}$ & $\mathrm{X}$ & $\mathrm{X}$ \\
\hline Uso eficiente da energia e conforto ambiental & $\mathrm{X}$ & $\mathrm{X}$ & $\mathrm{X}$ \\
\hline Qualidade do ar & & $\mathrm{X}$ & \\
\hline Manutenção do edifício & & $\mathrm{X}$ & \\
\hline $\begin{array}{l}\text { Uso sustentável de materiais, recursos e métodos } \\
\text { construtivos }\end{array}$ & $\mathrm{X}$ & $\mathrm{X}$ & $\mathrm{X}$ \\
\hline Gestão de resíduos & & $\mathrm{X}$ & \\
\hline Qualidade do ambiente interno & $\mathrm{X}$ & $\mathrm{X}$ & \\
\hline Inovação & $\mathrm{X}$ & & $\mathrm{X}$ \\
\hline Prioridade regional e desenvolvimento social & $\mathrm{X}$ & & $\mathrm{X}$ \\
\hline Gestão do canteiro de obras & & $\mathrm{X}$ & \\
\hline TOTAL DE CRITÉRIOS (12) & 8 & 9 & 6 \\
\hline
\end{tabular}

Fonte: FUNDAÇÃO VANZOLINI (2018); GREEN BUILDING COUNCIL BRASIL (2021); CAIXA (2021), modificado pelas autoras (2021).

Ao analisar o Quadro 1, percebe-se que os sistemas de certificação possuem critérios semelhantes, sendo o AQUA o que possui mais exigências, seguido do LEED, e o que possui menos exigências dentre os analisados é o Casa Azul CAIXA. Os itens "localização, transporte e qualidade urbana do entorno"; "uso eficiente da água"; "uso eficiente da energia e conforto ambiental"; e "uso sustentável de materiais, recursos e métodos construtivos" são critérios em comum entre os três sistemas de certificação analisados, fato que induz a importância desses itens para obtenção de selos de sustentabilidade. Porém, essas recomendações variam de acordo com o tipo de certificação almejada, o tipo de empreendimento e as características do local de inserção.

Percebe-se, entretanto, que a ausência de suporte às ações práticas nas obras leva as certificações a serem conhecidas apenas como uma estratégia de marketing que visam apenas a valorização comercial dos empreendimentos e falham nos pilares econômicos, sociais e ambientais (GOMES et al., 2016). Isso gera uma certa barreira para a aceitação das certificações sustentáveis pela indústria da construção civil.

Pardini (2009) afirma que a metodologia utilizada pelo sistema de certificação LEED é a mais aceita comercialmente, e norteia a maior parte das certificações brasileiras, visto 


\section{ounisul}

que embora seja uma certificação de origem norte-americana, é adaptável à realidade local, tanto no aspecto ambiental quanto social.

Entre as vantagens da certificação de um empreendimento, Grünberg et al. (2014) destacam o impulsionamento para busca de práticas mais sustentáveis para a construção civil, como melhora na gestão da obra e racionalização de materiais, além de ser um forte meio de atestar ao usuário o comprometimento ambiental do empreendimento. Essas são as mesmas vantagens já destacadas de um SGA.

Além disso, Pardini (2009) afirma que os custos de manutenção e operação de edifícios sustentáveis são menores comparados a edifícios tradicionais. Ou seja, os selos de certificação incentivam os investidores a construírem edifícios sustentáveis, pois traz uma gama de benefícios ambientais e socioeconômicos.

\section{3 papel do SGA para obtenção de selos ambientais em edifícios}

Para candidatar-se a um processo de certificação ambiental, o edifício precisa atender uma série de pré-requisitos que vão desde sua concepção à análise pós ocupação. Muitos desses requisitos só são possíveis se houver um SGA eficiente e integrado, pois, é necessário gerenciar a obra em todas as suas etapas. Conforme Gomes et al. (2016), o SGA é um processo metodológico que objetiva solucionar ou diminuir os impactos ambientais negativos como, poluição da água e do solo provenientes da grande quantidade de resíduos gerados, e uso indiscriminado de recursos naturais.

De acordo com Bueno e Rossignolo (2010), as metodologias das certificações exigem um bom sistema de monitoramento das atividades, ou seja, um bom SGA. Como o SGA faz parte de um sistema maior de gestão que estrutura e organiza uma empresa, é fundamental a clareza das metas a serem alcançadas para que o SGA possa gerenciar o processo em busca de atingir o propósito do empreendimento, seja ele conseguir uma determinada certificação de sustentabilidade, ou não (GOMES et al., 2016). Assim sendo, é necessária clareza na elaboração das metas e um estudo de viabilidade antes do início da construção, pois integrar um SGA na obra gera um acréscimo no orçamento. 


\section{ounisul}

Gomes et al (2016) realizaram um estudo de caso do SGA da construção de dois shopping centers que buscavam a certificação AQUA, sendo que um buscou o processo de certificação após o início da execução - denominado como empreendimento "A"; e o outro iniciou o processo de certificação desde o planejamento - denominado como empreendimento "B". Entre os critérios analisados por Gomes et al (2016), destaca-se o gerenciamento de resíduos da obra. O empreendimento "A" gerou aproximadamente $329,59 \mathrm{~kg} / \mathrm{m}^{2}$, sendo que o "B" gerou $134,81 \mathrm{~kg} / \mathrm{m}^{2}$. O edifício que teve um SGA desde o planejamento gerou aproximadamente $59 \%$ menos resíduos que o edifício que buscou o SGA após o início da execução. Além disso, Gomes et al (2016) destacaram que devido ao início tardio, o edifício "A" encontrou mais dificuldades na implantação do SGA, principalmente devido aos hábitos insustentáveis praticados pelos empregados, que precisaram ser conscientizados, diferentemente do edifício " $\mathrm{B}$ ", onde os hábitos sustentáveis foram implantados desde o início.

Como resultado final o shopping "A" recebeu avaliação do Selo AQUA como nível "bom", na categoria 3 - Canteiro de obras com baixo impacto; já o shopping "B" recebeu avaliação na mesma categoria como "excelente" em todas as fases do programa de certificação (Programa, Concepção e Realização) (GOMES et al., 2016). Isso confirma que seguir o Ciclo Plan-Do-Check-Act da ABNT (2015) faz diferença no resultado final do desempenho do processo de certificação, pois no empreendimento "A" a primeira fase - planejar, não foi aplicada antes do início da construção, e gerou prejuízo na avaliação do Selo AQUA.

A popularização do SGA gera mais edificações certificadas, e com isso mais experiência nesse processo de metodologia para obtenção dos selos ambientais que, consequentemente, diminui o custo adicional e aumenta a experiência da empresa construtora nesse processo (MUSTAFÁ, 2016). Ou seja, quanto mais experiência uma construtora tiver no SGA, menor será seu custo e maiores as chances de obtenção de certificações ambientais em seus edifícios. 


\section{ounisul}

Além disso, a certificação das obras são diretrizes para que o processo de produção e logística seja mais eficiente do ponto de vista econômico, e, portanto, atrai clientes conscientes (GOMES et al., 2016).

\section{CONCLUSÃO}

A certificação ambiental para edifícios tornou-se um grande atrativo para investidores, pois torna o empreendimento mais interessante comercialmente, expondo todo seu comprometimento com a sustentabilidade.

Os benefícios de uma edificação certificada são muitos, porém é necessário obedecer a vários critérios para a obtenção de algum desses selos. Muitos desses requisitos só podem ser alcançados com a implantação de um Sistema de Gestão Ambiental eficiente, pois é ele que gerencia a obra em todas as suas etapas.

Para obtenção de selos sustentáveis é ideal que o SGA tenha início ainda no processo de planejamento da obra, visto que desconsiderar essa etapa pode causar prejuízos muitas vezes irreparáveis, como o excesso de geração de resíduos.

Pode-se concluir que, embora haja dificuldades e custos iniciais para implantação, a busca por certificações ambientais para edifícios é viável e não é possível alcançá-la sem um SGA que integre todos os processos da obra, como preconiza a NBR ISO 14.001:2015. O ideal é que o SGA seja popularizado e passe a integrar a indústria da construção civil em todas as escalas, mesmo quando a obra não objetive a certificação. Para isso é necessário incentivo público, imposição de normas e fiscalização dos empreendimentos.

\section{Agradecimentos}

Ao Instituto Federal do Paraná (IFPR), pela concessão de bolsa PIBPós à primeira autora.

\section{Contribuições dos autores}

Os autores desse trabalho participaram da elaboração com as seguintes contribuições: 


\section{ounisul}

Ana Claudia Marangoni Batista Campana: realizou a conceituação, analise formal, metodologia, validação dos resultados e redação da minuta;

Dariane dos Santos Virgens Alvarenga da Silva: auxiliou na análise formal, na redação, revisão e edição;

Josiane Marlise Theis Aguirre: contribuiu na redação, revisão e edição;

Letícia dos Santos Marangoni Sígoli: contribuiu na redação, revisão e edição;

Máriam Trierveiler Pereira: orientou e supervisionou todo o trabalho.

\section{REFERENCIAS}

ABNT (ASSOCIAÇÃO BRASILEIRA DE NORMAS TÉCNICAS). Sistemas da gestão ambiental: requisitos com orientações para uso, NBR ISO 14001. Rio de Janeiro, 2015. 41p.

ASBEA (GRUPO DE TRABALHO DE SUSTENTABILIDADE). Guia sustentabilidade na arquitetura: diretrizes de escopo para projetistas e contratantes. São Paulo: Prata Desing, 2012. Disponível em: < https://www.caubr.gov.br/wpcontent/uploads/2017/05/asbea-sustentabilidade.pdf>. Acesso em: 21 de abr. de 2019.

BARRETO, I. M. C. B. N. Gestão De Resíduos Na Construção Civil. 1 ed. Aracaju: SENAI/SE; SENAI/DN; COMPETIR; SEBRAE/SE; SINDUSCON/SE, 2005. 28p.

BUENO, C.; ROSSIGNOLO, J. A. Desempenho ambiental de edificações: cenário atual e perspectivas dos sistemas de certificação. Revista Minerva, v. 7, n. 1, p. 45-52, 2010. Disponível em: <http://www.fipai.org.br/Minerva\%2007(01)\%2006.pdf>. Acesso em: 21 de abr. de 2019.

CAIXA. Selo Casa Azul CAIXA: Guia Selo Casa Azul +CAIXA_Julho2021.

Disponível em: < https://www.caixa.gov.br/sustentabilidade/negocios-sustentaveis/selocasa-azul-caixa/Paginas/default.aspx>. Acesso em: 20 de set. de 2021.

DAL FORNO, M. A. R. Fundamentos em gestão ambiental. Ed.1. Porto Alegre:

Editora da UFRGS, 2017. Disponível em:

<http://www.ufrgs.br/cursopgdr/downloadsSerie/derad108.pdf >. Acesso em: 21 de abr. de 2019.

FUNDAÇÃO VANZOLINI. AQUA-HQE. 2018. Disponível em: < https://vanzolini.org.br/produto/aqua-hqe/>. Acesso em: 20 de abr. de 2019.

GOMES, A. G.; MORAES, S. G.; MARINO, M. T. R. D.; FERNANDES, D. Sistema de Gestão Ambiental (SGA) e aplicabilidade do Selo AQUA: estudos de casos em empresas construtoras de shopping centers. Revista Tecnologia, v. 37, n. 1/2, p. 87110, 2016. Disponível em: <https://doi.org/10.5020/23180730.2016.V37.1/2.87-110>. Acesso em: 20 de set. de 2021.

GREEN BUILDING COUNCIL BRASIL. Documentos para download: Checklist LEED v4 BD+C. Disponível em:

<https://www.gbcbrasil.org.br/certificacao/certificacao-leed/tipologia-bdc/>. Acesso em: 20 de set. de 2021.

GRÜNBERG, P. R. M.; MEDEIROS, M. H. F.; TAVARES, S. F. Certificação ambiental de habitações: comparação entre LEED for Homes, Processo Aqua e Selo 


\section{sunisul}

Casa Azul. Ambiente \& Sociedade, v. 17, n. 2, 2014. Disponível em: < https://doi.org/10.1590/S1414-753X2014000200013>. Acesso em: 15 de set. de 2021.

HEYWOOD, H. 101 Regras básicas para Edifícios e Cidades Sustentáveis. São Paulo: Editorial Gustavo Gili, 2017. 272 p.

MAGDALENO, A. C. R. M.; NÓBREGA, M. J. R. Metodologias para Qualificação de Obras Civis Sustentáveis. Revista Augustus, v. 20, n. 40, p. 70-83, 2016.

MUSTAFÁ, Clarissa Mascarenhas. Sistema de Gestão Ambiental na construção civil: vantagens, dificuldades e mitos sobre sua implantação. Téchne, 2016. Disponível em:< https://techne.pini.com.br/2016/03/o-sistema-de-gestao-ambiental-na-construcao-civilvantagens-dificuldades-e-mitos-sobre-sua-implantacao/>. Acesso em: $21 \mathrm{de}$ abr. de 2019.

OLIVEIRA, J. A. C.; SPOSTO, R. M.; BLUMENSCHEIN, R. Ferramenta para avaliação da sustentabilidade ambiental na fase de execução de edifícios no Distrito Federal. Revista Gestão da Produção Operações e Sistemas, n. 2, p. 11, 2012. Disponível em: <https://revista.feb.unesp.br/index.php/gepros/article/view/527>. Acesso em: 20 de set. de 2021.

PARDINI, A. F. Contribuição ao entendimento da aplicação da certificação LEED e do conceito de custos no ciclo de vida em empreendimentos mais sustentáveis no Brasil. Campinas, 2009. 210 p. Dissertação (Mestrado). UNICAMP - Faculdade de Engenharia Civil, Arquitetura e Urbanismo.

RAMOS, M. A.; MARQUES, M. J.; MUYLDER, C. F.; BARROS, L. C. Sistema de gestão ambiental em uma empresa do setor de construção civil do município de Belo Horizonte. Apresentado In: XXXV Encontro Nacional De Engenharia De Produção: Perspectivas Globais para a Engenharia de Produção, Fortaleza: Enegep, 2015. Disponível em: < http://www.abepro.org.br/biblioteca/TN_STO_214_269_26975.pdf>. Acesso em: 22 de abr. de 2019.

SILVA, V. G.; SILVA, M. G.; AGOPYAN, V. Avaliação de edifícios no Brasil: da avaliação ambiental para avaliação de sustentabilidade. Ambiente Construído, v. 3, n. 3, p. 7-18, 2003. Disponível em:

<https://www.seer.ufrgs.br/ambienteconstruido/article/view/3491>. Acesso em: 20 de set. de 2021.

VECHI, N. R. G.; GALLARDO, A. L. C. F.; TEIXEIRA, C. E. Aspectos ambientais do setor da construção civil: uma contribuição para a adoção de sistema de gestão ambiental pelas pequenas e médias empresas de prestação de serviços. Sistemas \& Gestão, v. 11, n. 1, p. 17-30, 2016. Disponível em: < https://doi.org/10.20985/19805160.2016.v11n1.733>. Acesso em: 20 de set. de 2021. 